\title{
Ações educativas em saúde bucal com crianças com câncer: relato de experiência
}

\author{
Educational actions in oral health with children with cancer: experience report
}

Acciones educativas en salud bucal con niños con cáncer: informe de experiencia

Aluísio Ferreira Celestino Júnior ${ }^{1 *}$, Ana Carolina Carvalho dos Santos Milhomem¹, Luísa Helena Santos da Silva ${ }^{1}$,Bruno da Gama Gomes ${ }^{1}$, Rafaela Klautau Guerra ${ }^{1}$,Rebeca Antunes de Medeiros ${ }^{1}$, Victor Cezar Bendelaque Farias ${ }^{1}$, Yasmim Marçal Soares Miranda', Nicole Pantoja de Oliveira Santos ${ }^{1}$.

\begin{abstract}
RESUMO
Objetivo: Relatar ações de educação em saúde bucal desenvolvidas em uma instituição de apoio a crianças e adolescente portadores de câncer em um município da Amazônia brasileira. Relato de experiência: estudo descritivo do tipo relato de experiência que consistiu na utilização de metodologia ativa (Metodologia da Problematização através do Arco de Marguerez). As atividades privilegiaram estratégias lúdicas, valorizando a experiência dos participantes, visando a incorporação de hábitos saudáveis diante de situações críticas de saúde e de morbidade bucal. Em que pese as dificuldades individuais no enfrentamento da neoplasia maligna, mudanças importantes de comportamento para a adoção de práticas adequadas de autocuidado foram desenvolvidas no sentido de entender o significado desses cuidados no contexto geral de saúde e incorporálos em sua rotina. Considerações finais: Práticas de educação em saúde para grupos de alta vulnerabilidade devem valorizar estratégias que envolvam a participação efetiva. Nestas condições, o incentivo ao autocuidado e a compreensão da relação de causa e efeito que envolvem os determinantes de morbidade são relevantes. Realizar tudo isso em atmosfera agradável, permite o envolvimento e a compreensão de que prevenir comorbidades é concorrer para promoção em saúde, mesmo em condições gerais pouco satisfatórias.
\end{abstract}

Palavras chave: Saúde bucal, Educação em saúde, Metodologias.

\begin{abstract}
Objective: This paper report oral health education actions carried out in an institution supporting children and adolescents with cancer in a municipality of the Brazilian Amazon. Experience Report: A descriptive study of the experience report type that consisted of the use of active methodology (Marguerez Arc Problematization Methodology). The activities favored playful strategies, valuing the participants' experience, aiming at the incorporation of healthy habits in the face of critical health and oral morbidity situations. In spite of the individual difficulties in coping with cancer, important behavioral changes for the adoption of adequate self-care practices were developed in order to understand the meaning of this care in the general health context and to incorporate them into their routine. Final considerations: Health education practices for high vulnerability groups should value strategies that involve effective participation. Under these conditions, the encouragement of self-care and the understanding of the cause and effect relationship involving the determinants of morbidity are relevant. Accomplishing all this in a pleasant atmosphere allows for the involvement and understanding that preventing comorbidities is competing for health promotion, even under unsatisfactory general conditions.
\end{abstract}

Keywords: Oral health, Health education, Methodologies.

${ }^{1}$ Centro Universitário do Pará (CESUPA), Belém-Pará. *E-mail: celestinojr@yahoo.com.br 


\section{RESUMEN}

Objetivo: Informar sobre acciones de educación en salud bucal realizadas en una institución de apoyo a niños, niñas y adolescentes con cáncer en un municipio de la Amazonía brasileña. Informe de experiencia: Estudio descriptivo del tipo de informe de experiencia que consistió en el uso de metodología activa (Metodología de problematización del arco de Marguerez). Las actividades favorecieron las estrategias lúdicas, valorando la experiencia de los participantes, con el objetivo de incorporar hábitos saludables frente a situaciones críticas de salud y morbilidad oral. A pesar de las dificultades individuales para hacer frente a la neoplasia maligna, se desarrollaron cambios de comportamiento importantes para la adopción de prácticas adecuadas de autocuidado con el fin de comprender el significado de esta atención en el contexto general de salud e incorporarlos a su rutina. Consideraciones finales: Las prácticas de educación sanitaria para grupos de alta vulnerabilidad deberían valorar las estrategias que implican una participación efectiva. Bajo estas condiciones, el estímulo al autocuidado y la comprensión de la relación de causa y efecto que involucra los determinantes de la morbilidad son relevantes. Lograr todo esto en un ambiente agradable permite la participación y la comprensión de que prevenir las comorbilidades es competir por la promoción de la salud, incluso en condiciones generales insatisfactorias.

Palabras clave: Salud bucal, Educación sanitaria, Metodologías.

\section{INTRODUÇÃO}

Segundo o Instituto Nacional do Cancer-INCA (BRASIL, 2017) são estimados para o biênio 2018-2019, em torno de 420 mil casos novos de câncer no país. No público infanto-juvenil (0 a 19 anos), estima-se em cerca de 12 mil casos novos, dos quais 1.200 na região Norte, sendo este o menor número de casos entre as macrorregiões brasileiras. O maior número de casos é esperado para as regiões Sudeste e Nordeste com 5.300 e 2.900 respectivamente. Nos últimos anos, a incidência nesta faixa etária representa $3 \%$ de todos os casos.

Campos FAT, et al. (2018) afirmam que crianças e adolescentes sob tratamento antineoplásico têm risco elevado de desenvolverem diversos efeitos colaterais, dentre os quais mucosite, xerostomia, herpes simples, gengivite, candidíase. Tais efeitos decorrem em grande parte da quimioterapia. Tais fármacos, em geral, não atuam de forma seletiva, agem também provocando danos às células normais, em especial as de crescimento rápido como as imunológicas e as gastrointestinais como por exemplo as da cavidade oral (OLIVEIRA EL, et al., 2018).

O tratamento de neoplasias malignas demanda complexa e exaustiva sequência de cuidados como também resulta em eventuais reações colaterais já mencionadas, algumas que repercutem na cavidade oral. Por se tratar de pacientes que, normalmente, se encontram debilitados, eventos como esses representam incremento negativo a ser superado pela equipe de saúde em prol da qualidade de vida desses indivíduos (SILVA PLN, et al., 2016).

Dessa forma, orientar o próprio paciente e seu responsável/cuidador diante destas situações é de significativa relevância. Entretanto, mesmo para as orientações mais simples, os desafios podem representar dificuldades para sua correta aplicação na rotina de cuidados (SANTOS ALG, et al., 2018).

Os efeitos colaterais da terapia podem ser reduzidos se a equipe de assistência estiver atenta em fase bem preococe da doença e tomar as medidas adequadas minimizando-os. Em toda fase da doença, entretanto, deve se realizar e motivar o paciente para o autocuidado nestas condições. Isso previne outros agravos à saúde e concorre para um melhor quadro sistêmico necessário ao tratamento da doença, notadamente em crianças (PAKSERESHT M, et al., 2018).

Na região Amazônica, há parte considerável de crianças e adolescentes que reside em áreas remotas desta região que não dispõem de recursos para se manterem alojados nos locais onde estão os hospitais de referência onde devem receber tratamento. Isto representa séria dificuldade (VIEIRA AF, et al., 2017). 
Estes indivíduos, por sua vez, não devem permanecer indefinidamente em ambiente hospitalar sob cuidados de radioterapia, quimioterapia ou mesmo pós cirurgia, sob risco de desenvolverem infecções oportunistas aumentando ainda mais o risco de eventos adversos (MENDES ACMM, 2019). Por outro lado, suas condições gerais, frequentemente, os impedem de deslocamento para sua residência, em geral distante e penosa pelas condições de estradas e transporte nesta região, amiúde, precários.

Para minimizar estes impactos, a Casa Ronald McDonald Belém, uma instituição da sociedade civil, oferece acomodações bastante satisfatórias para crianças e adolescentes que vivem nestas condições. Tratase de uma instituição privada que dá apoio a crianças e adolescentes portadores de câncer (ROCHA LDJ, et al., 2015).

Foi realizado, neste trabalho, relato de experiência sobre um conjunto de ações de cunho educativo, voltado para a saúde bucal com crianças e adolescentes portadoras de câncer. Estas crianças e adolescentes permanecem albergadas nesta instituição, enquanto se submetem a tratamento em um hospital de referência na cidade de Belém, estado do Pará. Foi neste contexto que este trabalho foi realizado e que é objeto deste relato.

\section{RELATO DE EXPERIÊNCIA}

Como estratégia metodológica, foi adotada a Metodologia da Problematização com o Arco de Maguerez (BERBEL NAN, 2011), a qual permitiu construir uma estratégia de planejamento e intervenção no espaço de cuidado onde crianças e adolescentes permenecem albergados enquanto aguardam tratamento em hospital de referência de câncer na capital paraense.

A Metodologia da Problematização consiste em estimular o estudante a observar a realidade de modo crítico, possibilitando que o mesmo possa relacionar esta realidade com a temática que está estudando, propondo soluções para o problema identificado (BERBEL NAN 1995). O Arco de Maguerez, por sua vez compreende cinco etapas: (1) observação da realidade; (2) levantamento de pontos chave (3) teorização; (4) hipóteses de solução; e (5) intervenção na realidade.

Seguindo esta metodologia, um grupo de 34 estudantes de uma faculdade de Odontologia de Belém-PA com orientação de um docente escolheram a casa Ronald McDonald para o desenvolvimento das ações. A Casa Ronald McDonald é uma instituição internacional ligada ao Instituto Ronald Mcdonald que presta apoio a crianças e adolescetes portatores de neoplasia maligna na cidade de Belém e que em sua expressão local recebe o nome de Associação Colorindo a Vida, recebendo pacientes de 0 a 19 anos procedentes de vários municípios da região Amazônica.

$\mathrm{Na}$ etapa de observação do arco de Maguerez foi realizada visita à instituição e analisado o seu funcionamento. Através de apresentação oral e com auxílio de um video institucional, foi realizada exposição dialogada por uma das dirigentes da Casa Ronald McDonald. Ao final, os autores puderam participar com perguntas para esclarecimentos complementares, consubstanciando assim a primeira etapa do arco. Essa etapa foi complementada com visita guiada às instalações da "Casa". Verificou-se, por exemplo que a instituição conta com 35 apartamentos confortáveis privativos a cada paciente tem direito a um acompanhante, banheiro privativo, refeitório coletivo com dieta sob cuidados de nutricioniasta, área de lazer, capela ecumênica, lavanderia, brinquedoteca e auditório para sessenta pessoas. Tudo oferecido gratuitamente aos pacientes e seus acompanhantes.

A segunda etapa do arco relaciona-se ao levantamenteo de pontos chave relacionados ao(s) problema (s) observado(s). Verificou-se, por exemplo, que muitas crianças apresentavam cáries e que nem sempre realizavam o autocuidado com saúde bucal adequadamente. Apesar de não terem sido observadas queixas de mucosite durante as visitas, alguns pais relataram que seus filhos ja haviam apresentado mucosites em outras ocasiões. Dessa forma, os autores escolheram a temática voltada para prevenir o surgimento das enfermidades clássicas da saúde bucal como cárie e gengivites como também as mucosites que, eventualmente, podem surgir em decorrência da quimioterapia. 
Após essa etapa, os autores decidiram trabalhar com crianças na faixa etária de dois a cinco anos, além de seus pais e responsáveis. Outros discentes, atuaram com faixas etárias diferentes e com abordagem distinta.

Em relação à terceira etapa - Teorização - os autores buscaram em fontes de dados virtuais a produção científica que serviu de base para as etapas subsequentes do trabalho. Com outros grupos de estudantes que desenvolveram estratégia semelhante, foi realizada uma Mostra Científica cuja temática central foi o Câncer, apresentada na forma de paineis científicos e socializado com a comunidade acadêmica.

A quarta etapa - Hipóteses de solução - consistiu em elaborar um plano de ação voltado ao problema levantado. O plano de ação contempla as estratégias de intervenção e todos os recursos necessários à sua execução, ou seja, a fase de planejamento.

A quinta etapa - Intervenção na realidade - foi realizada através de uma oficina sobre saúde bucal com crianças de 2 a 5 anos de idade, a qual buscou orientações sobre higiene oral com demonstração em objetos figurativos como personagens infantis ("Urso Pooh" e "Tigrão"), macro escova, macromodelo. Posteriormente, as crianças foram divididas em grupos menores, tendo um estudante como supervisor de cada ação. Cada grupo possuía uma atividade específica, tais como: jogo de memória, jogo de trilha, dominó, além de uma ofina de desenho e pintura. Todas estas tecnologias educativas foram confeccionadas pelos autores e se remetiam aos cuidados com a saúde bucal apresentadas em circuito lúdico, oportunizando a participação de todas as crianças e adolescente nas diferentes atividades.

Nas visitas realizadas, verificou-se que algumas crianças, apesar da eventual indisposição, participaram das atividades. Para minimizar essa limitação na participação, as atividades lúdicas desenvolvidas eram intercaladas com modalidades que permitiam menor desprendimento de energia. Para aqueles que tinham membro amputado ou deambulavam com limitação ou em cadeira de rodas, adaptações à dinâmica da atividade foram necessárias para que a participação se desse de maneira favorável.

Com as ações desenvolvidas através da metodologia da Problematização com o Arco de Maguerez, foi possível envolver estudantes de odontologia numa temática que provavelmente se defrontarão na vida profissional. Compreender causas e consequências da entidade patológica câncer é uma habilidade regular de qualquer curso de odontologia, mas a vivência em campo de prática real onde as situações estão dadas, permite um envolvimento em busca de construção de estratégias sob bases de cuidados por evidência, mas também humana, ética.

A estratégia e a atmosfera criadas, seguramente, são favoráveis a uma melhor intervenção de orientação e cuidado necessários diante de situação muitas vezes dramática da doença de base. Morbidades orais decorrentes ou não do tratamento antineoplásico poderão ser melhor controladas com boa informação e atitudes em direção ao cuidado bucal tão necessário para a qualidade de vida diante da situação tão delicada apresentada pelas crianças e adolescentes acolhidos pela instituição.

As crianças e adolescentes de faixas etárias diferentes participaram de atividades semelhantes aos que foram direcionadas neste estudo, mas desenvolvidas por outros estudantes, as quais contemplaram orientações sobre os principais agravos em saúde bucal e as formas de prevení-los. A abordagem para estes participantes valorizou elementos de ludicidade tais como as desenvolvidas para o grupo de crianças menores, porém com linguagem e dinâmicas apropriadas às respetivas faixas etárias.

\section{DISCUSSÃO}

Segundo Santos MO (2018), o perfil de câncer em crianças e adolescentes no Brasil não difere muito dos dados encontrados em outros países, convergindo principalmente para o grupo de países considerados em desenvolvimento. A incidência mediana em seu estudo apontou 139,99 casos por milhão. Este mesmo estudo apontou ainda que, na faixa etária de 0 a 19 anos, tal como a do grupo dos participantes deste relato, destacaram-se as leucemias com percentual de $26 \%$ dos cânceres neste grupo, seguidos dos linfomas com $14 \%$. 
Para redução das taxas de mortalidade, aspectos relevantes se relacionam ao diagnóstico precoce e ao tempo de início de tratamento. Profissionais de saúde adequadamente qualificados para o diagnóstico são fundamentais, o que resulta em prognóstico mais favorável da doença. Os cuidados da atenção primária têm grande relevância e ao se investir mais intensamente no rastreamento da doença, os resultados são, geralmente, muito significativos na vida desses pacientes (FREIRE MND, et al., 2019).

A terapia oncológica, entretanto, é um desafio não somente no que se refere às chances de cura, mas ao que se relaciona aos efeitos colaterais e adversos que crianças e jovens são submetidos. Náuseas, mal-estar, vômitos, astenia, entre outros efeitos colaterais são frequentes, principalmente concernente à quimioterapia (SANTANA ME, 2017).

Em estudo realizado com crianças hospitalizadas com câncer, Barbosa et al. (2010) verificaram que as principais manifestações bucais destes pacientes sob tratamento oncológico eram mucosite, náuseas, vômitos, xerostomia e disgeusia. $\mathrm{Na}$ abordagem exploratória inicial com os pais das crianças e adolescentes na Casa Ronald McDonald verificou-se que, apesar de não haver relatos de mucosites nos momentos das visitas, este e outros achados estavam presentes em alguns desses indivíduos em ocasiões diferentes e foram relatados por seus pais/responsáveis.

As mucosites orais são achados clínicos bastante frequentes no tratamento oncológico e, apesar de não terem sido encontradas durante as visitas há que se estar atento a esse e outros achados que quando controlados adequadamente ou mesmo evitados, garantem melhor qualidade de vida durante a doença. A inclusão de laser profilático no protocolo do hospital de referência onde fazem tratamento, seguramente minimiza o surgimento dessas lesões. Em que pese não ser características desse estudo avaliar essa condição, vários estudos destacam a eficácia desta conduta profilática na prevenção e tratamento da mucosite induzida por quimioterapia e radioterapia (SANTOS JTL e FERNANDES NETO JA, 2019; WELTER AP, 2019).

Achados clínicos gerais e locais concorrem para indisposição física e falta de motivação para realizar o autocuidado com higiene bucal na rotina desses indivíduos, favorecendo o surgimento de comorbidades como cáries e doenças gengivais (COELHO JMF, et al., 2016). A equipe de cuidadores deve estar sempre atenta a intervenções preventivas e terapêuticas que minimizem isso. Apesar da vulnerabilidade de um indivíduo portador de patologia grave na infância ou juventude, há necessidade de continuamente motivá-los para medidas profiláticas frente a novos eventos patológicos orais e que, quando presentes, sejam solucionados ou minimizados (SANTOS JMS, et al., 2019).

Segundo Sales ASL, et al. (2016) medidas de proteção específica contra os agravos em saúde bucal conseguem dirimir alguns problemas, dentre os quais aqueles relacionados à falta de informação sobre causas, consequências, além dos cuidados preventivos e terapêuticos disponíveis. Por outro lado, medidas isoladas de educação e saúde nem sempre têm o impacto significativo ao longo do tempo. Essa é uma limitação deste trabalho. Para minimizá-lo, entretanto, foram incluídos pais e responsáveis no sentido de dar continuidade às orientações e supervisão desses cuidados.

$\mathrm{Na}$ experiência aqui relatada, foram incluídos pais e responsáveis com a preocupação de serrem também uma unidade de cuidado, além do paciente oncológico. Faz-se necessário que futuros profissionais estejam atentos ao fato de que os familiares são importantes em todo o processo do adoecimento de seu familiar. A família de paciente grave desempenha papéis importantes na vida do doente e precisa estar preparada em relação a realização de pequenos procedimentos preventivos, aos cuidados de rotina, mas também estarem bem emocionalmente (SOUZA LC e OLIVEIRA BLG, 2017).

Ao desenvolver com crianças e adolescentes atividades de orientação de cuidados preventivos sob uma atmosfera agradável, se utilizando de recursos lúdicos, é possível estabelecer melhor vínculo e favorecer a adesão ao próprio tratamento oncológico (TOLOCKA RE, et al., 2019). Jogos, brincadeiras, gincanas contribuem para melhor entendimento dos processos que enfrentam em sua rotina, permitindo que compartilhem suas dúvidas, medos, preocupações. É importante que nesse contexto de limitação, esses pacientes possam realizer seus papéis sociais (DIAS, PLM e SILVA IP, 2018). 
Atividades educativas como as desenvolvidas neste estudo têm demonstrado eficácia em relação a adesão ao autocuidado como também a criação de vínculo entre equipe de cuidadores e pacientes, além de facilitar a interação o que facilita a maior aceitação da proposta preventiva sob uma atmosfera pedagógica que valoriza a ludicidade. Essas intervenções, entretanto, a longo prazo, podem se diluir se não forem periodicamente reforçadas tanto pela equipe de cuidadores como pelos pais e/ou responsáveis (SOUZA GF, et al., 2019).

\section{AGRADECIMENTOS}

Agradecemos a direção da Casa Ronald McDonald Belém por ter recebido nossa instituição/autores para a realização desse trabalho em suas instalações com sua comunidade de pacientes e colaboradores.

\section{REFERÊNCIAS}

1. BARBOSA AM, RIBEIRO DM, TEIXEIRA ASC. Conhecimentos e práticas em saúde bucal com crianças hospitalizadas com câncer. Ciência \& Saúde Coletiva. 2010; 1(15):134-142.

2. BERBEL NAN. A Metodologia da Problematização com o Arco de Maguerez e sua relação com os saberes de professores. Semina: Ciências Sociais e Humanas, Londrina, 2011; 32(1): 25-40.

3. BERBEL NAN. Metodologia da problematização: uma alternativa metodológica apropriada para o ensino superior Semina: Cio Soc.IHum, 1995; 16 (2): 9-19.

4. BRASIL, Ministério da Saúde. Instituto Nacional do Câncer José de Alencar. Coordenação de Prevenção e Vigilância. Estimativa 2018: incidência de câncer no Brasil / Instituto Nacional de Câncer José Alencar Gomes da Silva. Coordenação de Prevenção e Vigilância. - Rio de Janeiro: INCA, 2017.

5. CAMPOS FAT, CARVALHO AKFA, CABRAL GMP, et al. Manifestações bucais decorrentes da quimioterapia em crianças. Ver Campo do Saber. 2018; 4(5): 136-149.

6. COELHO JMF, SANTOS BM, MIRANDA SS, et al. Percepção da condição bucal de pacientes sob tratamento oncológico em uma unidade de alta complexidade em Feira de Santana-BA. Rev. Saúde Col. UEFS, Feira de Santana, 2016. 6(2): 51-58.

7. DIAS PLM, SILVA IP. A Utilização do Brinquedo durante o Tratamento de Crianças com Câncer: Percepções da Equipe Multidisciplinar. Revista Brasileira de Cancerologia 2018; 64(3): 309-315.

8. FREIRE MND, RIBEIRO AVG, TORRES LA, et al. A importância do diagnóstico precoce no cancêr infanto-juvenil Rev. Mult. Psic.2019; 13(46) 44-45.

9. MENDES ACMM. Infeções oportunistas em doentes com neoplasias malignas sólidas Dissertação (Mestrado em Medicina) - Faculdade de Medicina. Universidad do Porto, Porto, 2019; 29 p.

10. NUNES AS, SANTOS RH, CELESTINO JUNIOR AF. A importância do laser profilático em pacientes oncológicos Revista Eletrônica Acervo Saúde, 2018; 16(sup): 1957-64.

11. OLIVEIRA EL, CABRAL GM, GALVÃO AKFC, et al. Mucosite: uma revisão sistemática. Rev Campo do Saber. 2018; 4(5) 16-34.

12. PAKSERESHT M, BARAZ S, RASSOULI M, et al, A Comparative Study of the Situation of Bereavement Care for Children with Cancer in Iran with Selected CountriesJ Pediatr, 2018; 6(2): 7253-7263.

13. ROCHA LDJ, SOUSA RIC, CELESTINO JUNIOR AF. Atenção em saúde bucal a crianças e adolescentes com câncer em uma casa de apoio na cidade de Belém, Pará - Brasil, 2015. Ver Paraense de Odontologia.2015; 1 (1): 12-23.

14. SALES ASL, CASSEB T, CELESTINO JÚNIOR, A, et al. Cuidados de Saúde Bucal na Comunidade Escolar do Núcleo Educacional Fiore. Rev Guará, 2016; 4(5): 17-28.

15. SANTANA ME, COSTA EGO, CORREAA ARS, et al. O cuidar em oncologia pediátrica: um estudo baseado no processo de enfermagem. Revista Destaques Acadêmicos. 2017; 9(3): 228-236.

16. SANTOS ALG, SANTOS NG, OLIVEIRA MPS, et al. Atividades lúdicas com crianças portadoras de câncer. Rev Cient Fac Educ e Meio Ambiente. 2018; 9(2): 74-85.

17. SANTOS JMS, SILVA MEB, ARAÚJO RJS, et al. Atividades lúdicas e educação em saúde com crianças hospitalizadas: um relato de experiência projeto resgatar GEPNEWS. 2019; 2(2): 616-623.

18. SANTOS JTL, FERNANDES NETO JA, CATÃO MHCV. Fototerapia no tratamento da mucosite oral: uma revisão de literatura. Arquivos em Odontologia. 2019; 55(1): 10-13.

19. SANTOS MO. Incidência, Mortalidade e Morbidade Hospitalar por Câncer em Crianças, Adolescentes e Adultos Jovens no Brasil: Informações dos Registros de Câncer e do Sistema de Mortalidade. Rev Bras de Cancerologia. 2018; 64(3): 439-440. 
20. SILVA PLN, XAVIER GC, OLIVEIRA VV, et al. Câncer Infantil Vivências de Crianças em Tratamento Oncológico. Enferm. Foco 2016; 7 (3/4): 51-55.

21. SOUZA DS, OLIVEIRA MFV, CORREA GM, et al, A enfermagem na promoção do autocuidado de higiene corporal em escolares da Amazônia: relato de experiência . Revista Eletrônica Acervo Saúde, 2019; 21(sup): e-570.

22. SOUSA GF, OLIVEIRA KDP, QUERIOZ SMD. Educação em saúde como estratégia para adesão ao autocuidado e às práticas de saúde em uma unidade de saúde da família. Rev Med. 2019; 98(1):30-9.

23. SOUZA LC, OLIVEIRA BLG, fatores psicológicos envolvidos no câncer infantil. Revista Uningá. 2017; 51(2): 77-83

24. TOLOCKA RE, CORRÊA RE, LIMA MM, et al. Que relação é esta? Licere. 2019; 22(1): 221-227.

25. VIEIRA AF, NEVES B, TONELLI SR. Perfil epidemiológico da leucemia linfoide nas regiões do Brasil. Revista unilus ensino e pesquisa. 2017; 14(37): 332-345.

26. WELTER AP, CERICATO GO, PARANHOS LR, SANTOS TML, RIGO L. Oral complications in hospitalized children during antineoplastic treatment. Journal of Human Growth and Development, 2019; 29(1): 93-101. 